\title{
Estudios de usuarios en archivos municipales: una aproximación teórico-práctica
}

\author{
Alfonso Rubio Hernández \\ Universidad del Valle (Santiago de Cali, Colombia)
}

\subsection{Resumen}

Se presenta un estudio de caso de estudio de usuarios en el ámbito de los archivos municipales, concretamente en el Archivo Municipal de Logroño (Comunidad Autónoma de La Rioja). Se parte del análisis de tres fuentes indirectas: el Libro registro de préstamos y consultas de usuarios internos y el Libro registro de ciudadanos y Fichero de investigadores. Se propone una metodología que abarca un estudio de usuarios completo y que atiende tanto al usuario interno (gestor administrativo) como al externo (investigador y ciudadano). Se apuntan primero algunas características de estos tres tipos de usuarios; y, después de analizados los resultados, se presentan propuestas de mejora del funcionamiento en relación a cada tipo de usuarios y a la información que solicitan.

Palabras clave: Archivos municipales. Estudios de usuarios. Control de préstamos y consultas. Gestores administrativos. Investigadores. Ciudadanos.

\subsection{Abstract}

A case study in municipal archives user studies is presented, developed in the Municipal Archive of Logroño (Autonomous Community of La Rioja, Spain). Through the analysis of indirect sources - the registry of loans and consultations of internal users, and the registry of the queries of researchers and citizens -, a methodology is proposed that includes a complete study of these three kinds of users. Finally, a set of improvements in the operation of the Municipal Archive regarding their users and their queries is suggested.

Keywords: Municipal archives. User studies. Loans and queries control. Administrative managers. Researchers. Citizens.

\section{Introducción}

El archivo es ante todo un centro gestor de información y todas sus tareas están orientadas a satisfacer las necesidades informativas de sus usuarios. Conocer la relación de estos usuarios con la información y el servicio de archivo que se la

Scire. $10: 1$ (en.-jun. 2004) 217-236. 


\section{8}

Alfonso Rubio Hernández.

proporciona - sus hábitos, perfiles, comportamientos, motivaciones, actitudes, opiniones, expectativas, deseos, necesidades, demandas y grado de satisfacciónresulta ser un "aspecto clave para el diseño de nuevas perspectivas y directrices metodológicas en la función informativa del archivo" (Navarro, 2000, p. 39).

Las actuaciones de los archivos en cuanto a la relación de sus servicios de préstamo y consulta con el empleo que de él hacen sus usuarios se suelen limitar al cumplimiento de cuestionarios estadísticos (1). A través de ellos se evalúa cuantitativamente la documentación servida, así como el número de interesados que hacen uso de ella. Pero sólo son resúmenes mensuales, trimestrales, semestrales o anuales que apoyan la realización de la memoria anual de los archivos. Son sumas de cifras (número de consultas, de préstamos, de documentos servidos, de investigadores, etc.) valiosas y objeto de posible análisis, pero cuya relación intrínseca no se explicita y que no descienden a describir el comportamiento de una variada tipología de usuarios. Sirviéndose también de esta información estadística, relacionándola entre sí y con otros tipos de información y pautas, la aplicación de los Estudios de usuarios al archivo podría servir para estructurar la interrelación entre documentación, archivero y usuario, y contribuir, en definitiva, a facilitar, flexibilizar o adecuar la correspondencia entre información y usuario.

\section{Estudios de usuarios}

\subsection{Estudios de usuarios en archivos}

Los estudios de usuarios surgen en el campo de la Bibliotecología como un área multidisciplinaria del conocimiento que, a partir de diferentes métodos de investigación, analiza fenómenos sociales referidos a aspectos y características de la relación información-usuario. Ciertamente, como pone de manifiesto la elaboración de las Normas Internacionales de Descripción Archivística -inspiradas en normas bibliográficas basadas en modelos de acceso a la documentación a través de sistemas de indización y recuperación por descriptores-, se hace necesaria la colaboración entre las distintas Ciencias de la Documentación e instituciones que se ocupan de ella, como pueden ser las bibliotecas, los archivos y los centros de documentación. En palabras de Alberch y Boadas (1991, p. 22):

Delimitar la personalidad diferenciada de los archivos respecto a las otras instituciones gestoras del patrimonio cultural no debe significar un obstáculo en el reconocimiento de la existencia de ciertos puntos de confluencia, y en consecuencia tiene que contribuir a la potenciación de una gestión más racional y eficaz. Es a partir del conocimiento y reconocimiento de las funciones y servicios que deben prestar los distintos equipamientos - especialmente archivos, bibliotecas y museos - cuando se podrán elaborar políticas de colaboración que permitan desarrollar fórmulas que comporten una gestión más integral del patrimonio.

Scire. $10: 1$ (en.-jun. 2004) 217-236. 
En concreto, se trataría de acercar el interés por el estudio de usuarios y adecuar sus prácticas, puestas en marcha en bibliotecas y centros de documentación en el ámbito anglosajón desde el primer tercio del siglo XX, a las tareas archivísticas. Si en España los estudios de usuarios todavía son recientes y están dificultados por la falta de instrumentos estadísticos generales y actualizados y por la escasez de condiciones en las bibliotecas, sus aplicaciones en el campo de la archivística son incipientes (2).

\subsection{Definición y objetivos de los estudios de usuarios}

Elías Sanz (1994, p. 31) ha definido los estudios de usuarios como "el conjunto de estudios que tratan de analizar cualitativa y cuantitativamente los hábitos de información de los usuarios, mediante la aplicación de distintos métodos, entre ellos los matemáticos (principalmente estadísticos) a su consumo de información". Y en un sentido similar se expresa Germà Iturrate (1993, p. 78): "Per a aquesta finalitat d'anàlisi de l'usuari d'arxius, l'arxiver hauria de comptar amb la collaboració de professionals de diverses disciplines: sociòlegs, psicòlegs, economistes, estadístics, etc." Sanz advierte de la complejidad que supone estudiar los hábitos de información de los distintos usuarios, debido a las variables que entran a formar parte del comportamiento de éstos a la hora de buscar o utilizar los recursos de información, y define algunos conceptos implicados en el estudio de usuarios como información, conocimiento, necesidad, deseo, demanda, uso y requerimiento de información. Son conceptos básicos trasladables al campo de la Archivística, como pueden ser también sus aplicaciones y la caracterización de los tipos de usuarios de información. Sin embargo, cuestiones como la metodología de recogida de datos (tipos de métodos), su análisis y sus representaciones a través de conceptos estadísticos y la evaluación de los recursos de información (tipos de evaluación e indicadores bibliométricos), planteadas por el Manual de Sanz, están completamente adaptadas al estudio de usuarios en bibliotecas. Aun así, muchas de sus aportaciones conllevan la posibilidad de ser aplicadas al usuario de archivo en su relación con el documento de archivo.

En definitiva, los objetivos generales de los estudios de usuario en los archivos coinciden con los de los sistemas de información en general, dentro de los cuales se encontraría el sistema de archivo formando parte de la estructura básica de una organización (3). Hernández (1997, p. 9-10) los ha enumerado de forma extendida y minuciosa, clasificándolos en cuatro grupos. En primer lugar, identificar las necesidades de información para la toma de decisiones y para la evaluación de la efectividad de un sistema o servicio de información, específicamente: determinar si un archivo o sistema de información está cumpliendo su propósito; determinar el nivel de éxito de programas y servicios; establecer prioridades entre programas y servicios; mejorar sistemas, servicios e instalaciones; ayudar

Scire. $10: 1$ (en.-jun. 2004) 217-236. 
al diseño, continuidad, modificación o discontinuidad de programas y servicios específicos; evaluar la adecuación de los documentos e instalaciones; resolver problemas específicos y superar deficiencias; apoyar demandas presupuestarias; justificar la existencia del sistema o servicio ante una institución mayor o a otros grupos de usuarios que apoyan el sistema; actualizar los objetivos; y solicitar sugerencias. En segundo lugar, analizar la interacción del usuario con el sistema, esto es: determinar el nivel de satisfacción del usuario y sus actitudes hacia los sistemas de información; identificar los éxitos y los fracasos de los usuarios; mejorar las relaciones públicas, determinar niveles de conocimiento de programas y servicios y ayudar a la formación de los usuarios; determinar patrones y niveles de uso y acceso; determinar la proporción de usuarios potenciales que son usuarios reales; facilitar la comprensión de la transferencia de la información; mejorar la equiparación del usuario y la información: minimizar el número de obstáculos entre usuario e información; determinar las prioridades del usuario; e identificar las poblaciones de usuarios, usuarios potenciales y no usuarios. En tercer lugar, identificar las características generales del usuario, específicamente: determinar los intereses del usuario, los estilos de vida, las opiniones, las actividades, las actitudes, las características sicológicas y demográficas; identificar nuevas tendencias y necesidades; estudiar el flujo de la información y los hábitos de uso de la documentación; e identificar fuentes de información a las que recurre la gente, tanto dentro como fuera de los archivos y sistemas de información. Y, finalmente, apoyar los estudios científicos y comparativos, facilitando la prueba de hipótesis o la conducción de estudios comparativos con el objeto de aislar variables causales.

\section{Determinación de los tipos de usuarios}

Los estudios de usuarios permiten conocer sus distintos hábitos de información, así como observar si los usuarios siguen, en general, unas pautas de comportamiento a partir de las cuales los podemos agrupar, con el fin de facilitar sus estudio y permitir aplicar sus resultados al diseño y funcionamiento de los servicios de información. Elías Sanz (1994) clasifica a los usuarios de bibliotecas en cuatro grandes grupos: el investigador y el docente, la industria, el administrador, planificador y político y el hombre de la calle. Los manuales de Archivística, por lo general, hablan de poner la información que los documentos de archivo albergan, al servicio de la administración, entidad o persona generadora del archivo, de los ciudadanos y de la investigación y la difusión cultural. Cruz Mundet (1994), en el capítulo dedicado a la función cultural de los archivos de su Manual de archivística, hace referencia a la tipología de usuarios como un elemento a considerar a la hora de planificar una política de información y atención primaria al usuario de archivo, y comenta la introducción de dos nuevos tipos de usuarios a partir de

Scire. $10: 1$ (en.-jun. 2004) 217-236. 
los años cincuenta: el aficionado y el estudiante. El autor presenta brevemente las características de estos dos tipos de nuevos usuarios; y, luego, en el apartado del marketing de archivos diferencia entre usuarios internos y usuarios externos, y entre estos últimos distingue cuatro grupos: los investigadores científicos, los aficionados, los estudiantes y los ciudadanos en general. Germà Iturrate (1993) clasifica a los usuarios de archivo en relación a siete grandes ámbitos: en relación al gran público, la enseñanza primaria, la enseñanza secundaria, al mundo universitario (estudiantes, investigadores, etc.), al mundo institucional (gestores administrativos, planificadores culturales, técnicos de la administración, etc.), al mundo del asociacionismo (asociaciones o entidades públicas o privadas) y en relación a las minorías (parados, pensionistas, minorías lingüísticas o culturales, etc.). En una descripción esquemática del tipo de usuarios, Alfred Mauri (1995, p. 100-101) distingue "l'usuari intern, vinculat al propi organisme del qual forma part l'arxiu, l'usuari investigador, l'usuari investigador en formació, l'usuari per a la formació professional y l'usuari ocasional o el públic general".

Por otra parte, ya dentro de nuestro ámbito concreto de estudio, según las pautas y procesos que sigue el servicio de préstamo y consulta del Archivo Municipal del Ayuntamiento de Logroño, su gestión de usuarios queda reflejada en tres instrumentos de control (4): Libro Registro de Préstamos y Consultas de Usuarios Internos, Fichero de Investigadores y Libro Registro de Ciudadanos. A partir de ellos se ha clasificado a los usuarios en dos grupos: internos y externos, y dentro de los externos se han distinguido a los ciudadanos y a los investigadores. Es la propia tipología de usuarios que establece el Archivo, sin poder descender a más niveles de agrupación (5).

Las incipientes aportaciones teóricas sobre estudios de usuarios en archivos se centran en sus servicios de referencia con el fin de conectar el archivo a la sociedad a través fundamentalmente de dos actividades: la consulta de los documentos por los usuarios y la difusión de sus fondos. Es por tanto el usuario externo quien recibe mayores atenciones y más concretamente, el investigador. Realmente, los estudios de usuarios en un archivo deben cumplir con el requisito de ocuparse de todos sus usuarios, y éste es el objetivo del presente estudio, estructurado en tres partes: usuarios internos, investigadores y ciudadanos.

Hasta ahora, las únicas evaluaciones estadísticas realizadas por el Archivo Municipal sobre su servicio de préstamo y consulta eran meramente cuantitativas, y se reflejaban en los indicadores de su memoria anual. Esta es la primera aplicación teórica y práctica de la disciplina Estudios de usuarios a la gestión del Archivo Municipal de Logroño; disciplina que, avanzamos, nos descubre numerosa y valiosísima información que antes permanecía secreta en las íntimas e insospechadas relaciones información-usuario. 


\section{Metodología}

Cuando emprendemos un estudio de usuarios debemos seguir los pasos de toda investigación (Gómez, 1996, p. 230-231): identificar los objetivos del estudio o nuestras hipótesis; analizar qué información será necesario obtener; recopilar la información disponible y diseñar un modelo de recogida de los datos de campo que requiramos; identificar los usuarios objeto de estudio y seleccionar la muestra adecuada; planificar la recogida de datos, los aspectos temporales, burocráticos o materiales necesarios; obtener la información; analizar los resultados para realizar las conclusiones; y, finalmente, laborar las propuestas de adopción de medidas concretas en el archivo.

Las fuentes de información sobre los usuarios son muy diversas. Podríamos agruparlas entre aquellas que cabe considerar directas, de campo - cuando obtenemos la información de lo que nos dicen los usuarios mismos - o indirectas - cuando aprovechamos datos factuales del archivo contenidos en la memoria anual, estadísticas o instrumentos de control. Las posibilidades son infinitas (6). Nos hemos limitado a las fuentes indirectas y dentro de ellas, a los tres instrumentos de control de préstamos y consultas de que dispone el Archivo. Todos son, por tanto, usuarios reales. Como queda dicho,

1. Tipo de usuario [interno / externo: investigador $y$ ciudadano]

\subsection{Características}

2. Tipo de muestra [Mensual / Anual]

3. Instrumento de control analizado [Libro Registro de Préstamos y Consultas de Usuarios Internos, Libro Registro de Ciudadanos y Fichero de Investigadores]

3.1. Caracteres externos

3.2. Contenido

3.3. Observaciones

4. Diseño de la base de datos

4.1. Actuaciones previas

4.2. Campos utilizados

5. Consultas [Tipo de consulta]

5.1. Resultados

5.2. Análisis de los datos

6. Propuesta de aplicaciones

Figura 1. Esquema de la metodología utilizada cada uno de los instrumentos nos ofrece un tipo distinto de usuario. El Libro Registro de Préstamos y Consultas de Usuarios Internos, el tipo "usuario interno"; el Libro Registro de Ciudadanos, el tipo "usuario externo: ciudadano" y el Fichero de Investigadores, el tipo "usuario externo: investigador". La metodología, aplicada en cada uno de los estudios, correspondiente a 
cada uno de los tipos de usuarios, es idéntica. Dependiendo de la cantidad y calidad de información contenida en cada uno de los instrumentos de control, se han analizado unos aspectos $\mathrm{u}$ otros, $\mathrm{y}$ con un determinado nivel de detalle $\mathrm{u}$ otro. El esquema de la metodología se presenta en la figura 1 (7).

Mostrar los resultados completos del desarrollo de esta metodología en el estudio de los tres tipos de usuarios indicados es extenso (8). Nuestro interés aquí es demostrar la validez de esta metodología a través del análisis de fuentes indirectas, así como la utilidad de aplicar los estudios de usuarios a los Archivos Municipales, para lo que la información proporcionada se centra en la caracterización del tipo de usuario estudiado y las propuestas de mejora.

\section{Estudio de usuarios internos}

\subsection{Características del usuario interno}

“¿Para qué se producen los documentos y por qué se conservan? En primer lugar, para la gestión administrativa de los asuntos corrientes, son el apoyo informativo para la toma de decisiones; asimismo, por el valor probatorio que les concede la ley. En este sentido el archivo tiene como misión proporcionar los documentos, datos y referencias para el desarrollo rápido y eficaz de las actividades de cada entidad" (Alberch y Cruz Mundet, 1999, p. 12).

En este sentido, los administradores del Ayuntamiento de Logroño, siendo los propios productores de la documentación, son también sus primeros usuarios. La conservación de los archivos siempre ha estado vinculada al ejercicio del poder. Disponer de su documentación y de su acceso en una institución pública como el ayuntamiento, en cierta medida, sigue siendo un privilegio para gobernar y administrar. El funcionario, como agente de la administración e intermediario entre los órganos de poder y el ciudadano, es el encargado de legitimar este poder a través de la información que contiene la documentación que él mismo, y a lo largo del tiempo, desde que existe la institución del Ayuntamiento, genera. Así, en esta histórica línea, no de derecho, sino de privilegio al acceso inmediato a la documentación por parte del funcionario como usuario interno - y comparado con el resto de usuarios - los externos, el funcionario sería el usuario más antiguo.

A continuación esbozamos algunas características o consideraciones generales de este tipo de usuario, el gestor administrativo, en su relación con el uso que hace del Archivo (9). Su relación con el mismo está legislada por el procedimiento administrativo y el reglamento del archivo. Todavía está sometido a hábitos inconvenientes, arrastrados de antes de que se hubiese implantado un sistema de archivo. Pesa la tradición idiosincrásica del funcionario español, cuya característica más evidente era la negligencia. Hay establecida una relación de jerarquía administrativo-archivero, mientras que con otros tipos de usuarios se establece

Scire. $10: 1$ (en.-jun. 2004) 217-236. 


\section{Alfonso Rubio Hernández.}

una relación de igual a igual. Desconoce la utilidad potencial de la documentación de archivo, y, especialmente, las interrelaciones entre las series documentales que forman el fondo documental de la institución para la que trabaja. Ignora el carácter orgánico del fondo y, por tanto, que la información deseada, si no se encuentra en el tipo documental que en concreto solicita, puede encontrarse en otro que desconoce. Adolece de falta de confianza en su relación con los servicios que presta el archivo: "L'usuari inter sempre ha estat el més desatès de tots aquells que utilitzen l'arxiu. Per ell, l'arxiu solament és un mitjà per arribar després de tot un procés a una finalitat administrativa. Però un mitjà oblidat, abandonat i negligit" (Iturrate,1993, p.86). Generalmente sus peticiones o búsquedas se limitan al dato concreto y puntual, y su nivel de exigencia es el de la respuesta inmediata

\subsection{Propuesta de aplicaciones}

Los resultados y conclusiones que se obtengan a partir de la realización de estudios de usuarios, pueden tener una gran aplicación en muchas de las tareas vinculadas al mundo de la información y la documentación. [...] Estos estudios se deben realizar de una forma periódica, con el fin de detectar los cambios en los hábitos de información que se vayan produciendo en los usuarios, e introducir esos cambios en los centros para que sean capaces de responder de una manera eficaz a las nuevas necesidades. (Sanz, 1994, p. 31-32)

A la vista de nuestros resultados, a continuación desglosamos algunas de las posibles aplicaciones concretas que podrían llevarse a cabo en el Archivo Municipal de Logroño en su relación con los gestores administrativos de su plantilla: diseño y confección de instrumentos de control impresos o automatizados, uniformados y adecuados a cada uno de sus objetivos, completos y oficializados; establecimiento y unificación de criterios entre el personal del archivo a la hora de atender al usuario y registrar los préstamos y consultas; siendo lunes y jueves los días de la semana con mayor movimiento en el archivo, procurar contar con el total de recursos humanos en esos días; confeccionar instrumentos de descripción con mayor nivel descriptivo o particularizados para los tipos documentales más solicitados - licencias de apertura, licencias de obra, plusvalías y expedientes de planeamiento-; adecuar los instrumentos de descripción a las necesidades particulares de los servicios y tipos documentales que demandan; mantener durante más tiempo las plusvalías en el archivo de oficina - pues destacando sus peticiones del resto de tipos documentales, descongestionaría el servicio de préstamo y consulta del archivo-; habilitar espacios exclusivos para la consulta de los usuarios internos y no compartirlos con los investigadores en su sala; conocer los documentos que no han tenido ninguna demanda para realizar posibles expurgos o cambiarlos a depósitos menos frecuentados; acercar las unidades de instalación en depósito de los tipos más consultados a la sala de consulta, reduciendo el esfuerzo y el tiempo dedicado a la consulta; acrecentar las relaciones de confianza

Scire. $10: 1$ (en.-jun. 2004) 217-236. 
con el personal de Gestión e Inspección Tributaria y Urbanismo, los servicios que más documentación solicitan y facilitar los instrumentos de descripción incluso en sus propias dependencias; buscar las causas de los servicios que no utilizan el archivo con el fin de posibilitar, en caso de necesidad, la realización de cursos que den a conocer la potencialidad de la documentación de archivo y los cauces de acceso; determinar cómo se están cumpliendo los objetivos del archivo y el grado de satisfacción del usuario interno, para cambiar o mejorar su política de actuaciones; y, finalmente, comparar las prioridades del servicio de préstamo y consulta del archivo con el resto de sus actividades.

\section{Estudio de usuarios externos}

\subsection{Características del tipo de usuario externo "investigador"}

Como archiveros, no debemos pensar que trabajamos aisladamente al servicio de pequeños públicos esotéricos, sino como parte integrante de un entorno cultural del que depende la supervivencia de la humanidad. La cultura es una palabra casi imposible de definir, pero cabe entenderla en este contexto como lo que nos hace más acabados y más capaces de interactuar plenamente con los demás en nuestra aldea mundial. (Taylor, 1984, p. 3)

En cierto sentido, todas las funciones y tareas del archivero, están enfocadas a constituir un servicio que directa o indirectamente facilite los objetivos de los usuarios. Como el mismo autor (ibídem, p. 4) nos indica:

[...] si no hay usuarios (entre ellos nosotros mismos), los documentos y la información que contienen tendrán sólo un potencial, una energía tensada, la cual se descarga por medio de la interacción dinámica de la intervención humana con: a) el portador, por ejemplo, el pergamino, el papel o la cinta magnética; b) la configuración y la forma de los símbolos (alfabeto cuneiforme, pictográfico o fonético); y c) la propia información, definida en general como un contenido.

Siguiendo a los autores anteriormente citados en el presente trabajo, al estudiar el Fichero de Investigadores del Archivo Municipal del Ayuntamiento de Logroño estaríamos, según Cruz Mundet (1994), ante los usuarios externos investigadores científicos, los aficionados y los estudiantes; según Iturrate (1993), ante el mundo universitario(estudiantes, investigadores, etc.); y según Mauri (1995), ante el usuario investigador, el investigador en formación y el usuario de formación profesional.

Siendo el investigador el usuario más diverso y de mayor exigencia en sus requerimientos y dada la complejidad de la interacción entre archivero, documentación, información e investigador tan grande, son muchas las consideraciones que deben tenerse en cuenta en su estudio. En primer lugar, la información es una noción abstracta y generalizada, con una tecnología igualmente abstracta y generalizada que la gobierna. Pero lo que abruma a la sociedad es la ingente cantidad

Scire. $10: 1$ (en.-jun. 2004) 217-236. 


\section{Alfonso Rubio Hernández.}

de detalles concretos que hay que dominar y entender en la información. De ahí la tensión entre el archivero y el usuario, entre los métodos que utilizan los principios archivísticos de procedencia y orden original y la localización mediante una indización muy específica por temas. En segundo lugar, la captura de una información idónea por el investigador constituye una operación sumamente compleja. Hay una confrontación del usuario con el contenido del documento que solicita, y en ella su experiencia no será idéntica a la de otro usuario. Sus experiencias variarán en función de su formación previa, sus antecedentes y de la finalidad que vaya a darle a la información. En tercer lugar, las necesidades y comportamientos de los investigadores varían según sus disciplinas, los conceptos de valor que aplican a la información y sus funciones en la sociedad. El archivero debe distinguir entre el acceso intelectual y el hecho de facilitar los documentos físicamente. Debe procurar que el usuario se sienta atendido en sus necesidades, pero ¿cuáles son esas necesidades y en qué medida es el conservador, más que el usuario, el responsable de satisfacerlas? En cuarto lugar, hay que considerar las implicaciones físicas del creciente uso académico de los archivos: salas de lectura, métodos reprográficos, préstamo interarchivístico, atención del personal de archivo, técnicas de expurgo, infraestructura de conservación, etc. Según Michael Roper (1982, p. 339):

[...] cualquier aumento en el uso de los archivos impone una mayor presión en cuanto a las salas de lectura y a los sistemas de producción de documentos. Aun donde el creciente uso académico no es el único factor que contribuye a tal aumento en la presión... los usuarios académicos tienden a visitar más frecuentemente los archivos y a consultar mayores cantidades de documentos en cada visita que los usuarios.

En quinto lugar y finalmente, hay que considerar la información y orientación al usuario investigador, que podría clasificarse en (Franz, 1981, p. 306) tres grandes clases: a) información general - directorios internacionales, nacionales y regionales, bosquejos y folletos sobre oficinas de archivos particulares, publicaciones periódicas y medios públicos como instrumentos de información archivística-; b) preparación de la investigación archivística - guías e inventarios generales, guías tradicionales de localización de archivos, información orientada por temas - ; y c) los efectos en el usuario - consultas y servicios para el usuario en el archivo, estadísticas-.

Aquí, únicamente nos centraremos en señalar algunas características sobre este tipo de usuario externo, el investigador. Podríamos particularizar sus características según el sector al que pertenecen - investigadores profesionales, en formación, estudiantes, etc. - , pero - por la falta de algunos campos informativos en los registros que componen el Fichero de investigadores, que podrían habernos ofrecido más datos de contraste de dichos sectores - nos hemos limitado a extraer algunas características del investigador en general: diversidad formativa

Scire. $10: 1$ (en.-jun. 2004) 217-236. 
- historiador, sociólogo, economista, geógrafo, científico, etc.-; preparación archivística; especialización en cuanto a la documentación que busca; familiaridad con el ambiente de los archivos y el uso de la sala de investigadores, por lo que su predisposición y confianza a la hora de enfrentarse al documento es mayor (10); y asiduidad. Es, pues, un usuario formado y preparado para utilizar los instrumentos de descripción del archivo. No necesita orientación pedagógica, sino puramente informativa de los fondos. No es un usuario pasajero o fugaz. Al menos temporalmente en el transcurso de sus investigaciones, sus visitas suelen ser frecuentes y consultan mayores cantidades de documentos en cada visita que el resto de usuarios. En el campo universitario suele haber interacción archivo-universidad. Este tipo de usuarios está en crecimiento, tanto en cantidad como en el nivel de sus exigencias en aras a un mayor rigor histórico-científico. Finalmente, y como ocurre con otros tipos de usuarios personales, sus datos personales, que se registran en la ficha de investigadores, están sujetos a la Ley Orgánica 15/1999 de 13 de Diciembre de Protección de Datos de Carácter Personal

\subsection{Propuesta de aplicaciones}

Entre las posibles aplicaciones que podrían llevarse a cabo en el Archivo Municipal de Logroño en su relación con sus investigadores, está el completar con más campos de interés las fichas modelo o registros del fichero de investigadores: número de registro de la ficha, nacionalidad, edad, fecha de inicio y conclusión de la investigación, tipologías documentales consultadas, objeto del estudio - tesis, doctorado, trabajo de investigación-, publicación - artículo, monografía, otros - y título. Con el campo de tipologías documentales consultadas, podríamos analizar de manera relacionada qué tipologías son las más consultadas por cada una de las profesiones de los investigadores y se podrían adecuar los instrumentos de descripción a las necesidades demandadas. Otra línea de trabajo es automatizar los datos contenidos en el fichero de investigadores para obtener estadísticas sobre las tendencias que en el terreno de la investigación se dan, en nuestro caso, en la Comunidad Autónoma de La Rioja o en la ciudad de Logroño. Estos datos facilitarían la gestión política de la investigación, revelando qué se está investigando y en qué áreas - humanísticas, científicas, etc.-. En tercer lugar, procede reforzar las relaciones de confianza entre el archivero y el investigador para conocer de qué manera está funcionando el servicio de referencia del archivo, y medir el grado de satisfacción del usuario en relación con las atenciones del archivero, la sala de investigadores, la biblioteca auxiliar de la sala, los servicios de reprografía, los horarios de apertura, la adecuación de la documentación solicitada con la información necesaria, etc. Si el usuario frecuenta con asiduidad el servicio del archivo, la relación entre el archivero y el investigador se puede particularizar y acrecentar para conocer los objetivos reales

Scire. $10: 1$ (en.-jun. 2004) 217-236. 
del investigador, qué información necesita realmente: Tal vez se estén solicitando unas determinadas tipologías documentales y no sean las idóneas, o haya otras con información relacionada. En esta dirección, se podrían elaborar cuestionarios o encuestas a investigadores para determinar cómo se están cumpliendo los objetivos del archivo y poder tomar decisiones concretas para mejorar las políticas de actuaciones (11). Finalmente, sería posible comparar las prioridades del servicio de investigadores, respecto a las del servicio de consultas a ciudadanos y el de préstamos y consultas al personal interno, así como, posteriormente, con el resto de las actividades del archivo.

\section{Estudios de usuarios externos: ciudadanos}

\subsection{Características del tipo de usuario externo "ciudadano"}

Progresivamente los archivos van dejando de ser el patrimonio de minorías eruditas para convertirse en ilustración y en medio de cultura. Hacen tránsito de un estadio pasivo, centrado en la conservación de los documentos y su disponibilidad para un público selecto, a un papel más activo de mediación cultural. Por múltiples canales, en verdad modestos todavía, el gran público comienza a entrever sus recursos. Pero, ¿no faltará mucho por hacer en el empeño de familiarizar a todas las riquezas de un sector desconocido o no apreciado del patrimonio cultural de su país? (Berche, 1982, p. 367).

Según el Libro Registro de Ciudadanos del Ayuntamiento de Logroño, nos encontraríamos ante el usuario que podríamos denominar ciudadanos en general, el gran público o el usuario ocasional o el público general. Siguiendo a la misma autora (ibídem), todavía en los años ochenta, la idea de que los archivos se activaran en beneficio de un gran público no era unánime entre todos los archiveros y teóricos de la archivística. Se veía que dicha acción conllevaba dos aspectos: a) la difusión de los recursos culturales en favor de los interesados; y b) la publicidad en favor de los archivos. Y ante ellos, aparecían dos actitudes contrapuestas entre los archiveros. Por un lado, algunos se apasionan por las actividades de formación y de enriquecimiento intelectual de un público profano, poniendo en evidencia el papel de los archivos. Y por otro lado, otros se preguntan por la utilidad de influir en un público más numeroso que el que frecuenta normalmente las salas de lectura o consulta de los archivos. Dedicados tradicionalmente al papel administrativo y científico de los archivos, éstos últimos tienden a menospreciar el lado vulgarizador y publicitario del servicio de archivo.

Como decíamos y así vuelve a exponerlo Claire Berche (ibídem, p. 375), "la importancia del papel cultural de los archivos exige ciertamente medios de personal, créditos y material, pero implica también que el grupo de funcionarios de los archivos acepte que vuelvan a cuestionarse algunos de sus conceptos, seguir las modalidades actuales y los gustos del público, iniciarse en las nuevas técnicas

Scire. $10: 1$ (en.-jun. 2004) 217-236. 
que le son a menudo extrañas y a veces no son de su agrado, y practicar una colaboración activa en el complejo de los organismos de vocación cultural"

El responsable de archivo es el intermediario entre unos fondos documentales y el pueblo que los ha generado. En el contexto de la acción cultural contemporánea, los archivos deben ayudar a la formación del criterio. Hacen comprender la finalidad de nuestras instituciones nacionales, regionales o locales; contribuyen a la afirmación de una identidad y permiten aprehender la historia de los pueblos, sus leyendas, costumbres y tradiciones que van vivificando la historia de ayer y la de hoy. Las tendencias y experiencias actuales llevadas a cabo en cuanto a animación cultural y publicidad en la relación archivos/gran público, pueden concretarse de manera generalizada en las siguientes: museos de archivo, exposiciones, actividades educativas, publicaciones, emisiones radiales o de secuencias televisadas sobre los recursos de archivos, jornadas de puertas abiertas o semanas de archivo, etc.

Centrándonos en las características generales de este tipo de usuario, el ciudadano, en su relación con el uso que hace del Archivo y tomando como ejemplo este estudio aproximativo concreto basado en el Archivo Municipal de Logroño, apuntamos algunas de las principales. Acostumbra a ser una persona indeterminada, de edad variable y de un nivel cultural impreciso (12). No acude con regularidad al archivo, sino ocasionalmente. Suelen ser personas que, sin participar de manera estable en acontecimientos culturales, tienen sensibilidad por su patrimonio cultural, del cual el documento forma parte. Acude, sin embargo, con asiduidad el consultor que busca solucionar un problema puntual y en esta línea podemos decir que es un usuario con sentido práctico. En numerosas ocasiones el usuario acude por primera vez y desconoce el procedimiento de las consultas, las características de los fondos documentales que el archivo conserva, las tipologías documentales a consultar y la sala de consulta donde debe desenvolverse. Es un usuario que necesita orientación y mayor atención que cualquier otro usuario.

\subsection{Propuesta de aplicaciones}

Analizado el Libro registro de ciudadanos, proponemos algunas posibles aplicaciones concretas que podrían materializarse en el Archivo Municipal de Logroño en cuanto a la relación de este tipo de usuario con el servicio del archivo, pues realmente el promedio que efectivamente se da de siete consultas diarias de documentos, casi está exigiendo una más adecuada o cuidada atención al ciudadano. Convendría, en primer lugar, proceder al diseño y confección de un Libro registro de ciudadanos impreso y automatizado, uniformado y adecuado a las exigencias estadísticas y la realización de estudios de usuarios para proveer mejoras en el servicio. Habría que delimitar claramente qué usuarios se incluyen en el Fichero de investigadores y qué usuarios se incluyen en el Libro registro de

Scire. $10: 1$ (en.-jun. 2004) 217-236. 
ciudadanos, así como sus respectivos espacios en la sala de consulta. Convendría mejorar la atención del servicio de archivo a este último tipo de usuario, mayormente profano, y personalizar las relaciones a través del diálogo, pues sus hábitos de información son los más complejos. Aquí, los conceptos mencionados al comienzo del presente estudio, a los que hace referencia Elías Sanz (1994), de necesidad, deseo, uso de información, etc., y que están implicados de forma decisiva en el estudio de usuarios, se hacen más difícil de desentrañar. Como dice Taylor (1984, p. 14-16).

Por ser todo usuario un individuo complejo, la relación con la información será multidimensional. Como dice Holmer Hall: "Cuando prospera la dicotomía, la simplificación excesiva pretende negar el derecho a ser diferentes". Tenemos que saber lo más posible sobre los usuarios y no simplemente cuáles son sus campos de interés inmediato. Debemos pensar también más en los usuarios eventuales al elaborar nuestros medios de localización. ¿Qué buscan? ¿Ha de servir un solo medio de localización para todos los tipos de usuarios?...los usuarios propenden a ordenar sus "necesidades" en "listas de compra" fragmentadas, mientras que sus auténticas necesidades sólo pueden determinarse a partir de una mayor comprensión del usuario como persona.

En el caso del Archivo del Ayuntamiento de Logroño, siendo los Expedientes de obras particulares una serie documental tan consultada, sería necesario elaborar instrumentos de descripción específicos para esta serie, con un nivel de descriptivo más amplio que el de inventario. Sería un instrumento valioso y práctico. Por fin, convendría elaborar cuestionarios para ciudadanos usuarios con el fin de conocer cómo se están cumpliendo los objetivos del archivo y poder contar con criterios a la hora de tomar decisiones, así como evaluar las prioridades del servicio de atención al ciudadano respecto al resto de las actividades desempeñadas por el archivo.

\section{Conclusiones}

"La expansión de los roles de archivo, el aumento de las colecciones de archivo, los nuevos tipos de archivos, más y mejores guías de localización, nuevos campos de interés científico, una mayor conciencia de las posibilidades que la investigación archivística brinda a la administración, al mundo académico y al público en general, menos horas de trabajo que dejan más tiempo libre y una última en orden pero no en importancia, un casi sorpresivo renacimiento del interés histórico, todo esto ha hecho que se llenen los salones de los archivos y que se multiplique el número de consultas por escrito" (Franz, 1981, p. 307). Uno de los efectos de los estudios de usuarios aplicados a cualquier sistema de información, debe ser la progresiva supresión de las barreras que separan a los profesionales relacionados con la gestión de la información de sus usuarios. El impacto de las nuevas tecnologías, cada vez más, está cambiando la naturaleza del trabajo, el

Scire. $10: 1$ (en.-jun. 2004) 217-236. 
cual se está caracterizando por la instantaneidad, la descentralización y la colaboración o intercambio de información. En el caso que nos ocupa el intercambio de información se produce en un doble plano: entre los propios profesionales y entre profesionales-usuarios. Todo esto repercute en el destinatario final -el usuario - , cada vez más familiarizado con estos medios y que espera respuestas más ágiles y acordes con los tiempos.

En este sentido, limitándonos al ámbito de los "usuarios internos" del Ayuntamiento de Logroño, algunas series documentales como los Padrones tributarios, están reproducidos en dos tipos de soporte: papel e informático. Los padrones en soporte papel se conservan en el Archivo, pero los padrones en soporte informático, son consultables a través del sistema informático por todos los usuarios internos con acceso a ellos, disminuyendo así la presencia física del solicitante en el Archivo. La mayor y mejor relación usuario interno-archivo a través de las nuevas tecnologías y dentro de un mismo sistema de organización, como resulta ser el Ayuntamiento de Logroño, sería un primer paso factible que simplificaría el intercambio de información entre el servicio del Archivo y el gestor administrativo. En cuanto a la reacción del archivero ante el comportamiento de los "investigadores", parecen oportunas, a manera de conclusión, las reflexiones de Hugh A. Taylor (1984, p. 18):

Los archiveros necesitan saber muchas más cosas sobre el modo en que los científicos de las disciplinas citadas llevan a cabo sus investigaciones y acrecientan el universo del saber. Necesitamos una ciencia del crecimiento del saber, una ciencia de la información que guarde con el almacenamiento y la localización o recuperación la misma relación que la física con la ingeniería... Una ciencia de los archivos que formulara preceptos a partir de la interfaz de los medios de las fuentes de investigación con los estudios interdisciplinarios podría incitarnos a crear los efectos que necesitamos nosotros y los usuarios, en vez de reaccionar constantemente ante unas causas engendradas por las necesidades específicas de los usuarios. Con ello, habríamos de interesarnos más no sólo por el contenido de los documentos (así es como reaccionamos ante los usuarios) sino también por la utilidad posible de los mismos, como parte integrante de las estrategias de adquisición de originales y de copias e introduciendo elementos críticos a la vez que descriptivos en los medios de localización".

Y del interés del "gran público" por los archivos (Berche, 1982, p. 369):

[...] da hoy testimonio su gusto creciente por la historia, en la medida en que la parte que se le entrega toca con los programas escolares. Éxitos de emisiones televisadas (películas y debates) sobre temas históricos, difusión de obras o de revistas culturales accesibles a todos, a cargo de universitarios conocidos, publicaciones vendidas hasta en los supermercados, multiplicación de las sociedades históricas locales, desarrollo de la genealogía, tantos signos que traducen, según expresión un poco trajinada, el deseo de "reencontrar sus raíces" en una civilización en cambio permanente, la admiración exagerada por las civilizaciones tradicionales y la vida cotidiana de antaño.

Scire. $10: 1$ (en.-jun. 2004) 217-236. 


\section{2}

¿Por qué, en este movimiento, no intentar proveer al público de instrumentos que le enriquezcan sus conocimientos, que le enseñen a utilizar nuestros recursos?

En definitiva, el archivo orientado al usuario es el centro que adecua sus espacios, sus documentos, las normas de uso o la distribución de sus actividades técnicas a las necesidades de sus usuarios. A promover esta orientación contribuyen los estudios de usuarios con los objetivos de mejorar la accesibilidad, disponibilidad y conocimiento de los fondos documentales, reducir el tiempo de localización y acceso a la información y a los documentos, e ir adaptando el archivo en cada momento a las demandas de información cambiantes de los usuarios, utilizando los recursos informativos y tecnológicos disponibles para conseguirlo.

\section{Notas}

(1) La estadística de archivos, bibliotecas y museos se reorganizó en la orden de 23 de diciembre de 1959 de la Presidencia del Gobierno publicada en el Boletín Oficial del Estado de 22 de enero de 1960. A ella se adjuntan cuestionarios modelos que deben diligenciar los archivos; y, en concreto el cuestionario A2 (Resumen de actividad) recoge campos informativos sobre el movimiento de fondos, la documentación servida, los investigadores y la acción cultural del archivo. El modelo A3 es un ejemplo de Ficha de investigador. Según su Artículo $1^{\circ}$, "la mencionada estadística alcanzará a los archivos y bibliotecas regulados por el Decreto del Ministerios de Educación Nacional de 24 de julio de 1947, [...] todos los Archivos y Bibliotecas de carácter civil de la Administración central, provincial y local, los de las Delegaciones permanentes del Estado en el extranjero y cuantos de la misma naturaleza se declaren de interés nacional o local para el estudio y conocimiento de la Historia y cultura patrias". En cuanto a los plazos en que deben diligenciarse los cuestionarios, el Anexo de la orden dice: "Los cuestionarios modelo A2, B2 y M2 se dilligenciarán cada trimestre por los archivos, las bibliotecas y los museos. Los cuestionarios modelos A3 y M3 se diligenciarán con referencia a los investigadores o copistas que hubiesen iniciado su trabajo cada trimestre" (Legislación Básica, 1982).

(2) Un estado general de la cuestión, centrado en el servicio de referencia archivístico, nos lo ofrece Navarro (2000). Sus referencias bibliográficas abarcan el panorama internacional y son escasas en cuanto al hispanoamericano, corroborando lo que dice el texto.

(3) "Hay que advertir que restringimos el universo de organización a las instituciones con una personalidad jurídica reconocida, pública o privada, del tipo de empresas, colectivos sociales u organismos autónomos dependientes o incluidos dentro de la Administración Pública" (Esteban, 1997, p. 288).

(4) Utilizamos la denominación de servicio de préstamo y consulta como una actividad más integrada en las funciones del Archivo. No es un servicio o departamento independiente que forme parte de la estructura orgánica del Archivo, como lo es el Departamento de Referencias del Archivo Histórico Nacional ( Díez de los Ríos, 1996, p. 235): "El área que atiende los aspectos de difusión es el Departamento de 
Referencias. Su objetivo principal es poner al servicio de la sociedad la documentación que se conserva en el archivo y está organizada".

(5) Sólo en el estudio de investigadores podemos saber la profesión del usuario y descender al análisis de los mismos por profesiones. Sin embargo, el Libro Registro de Ciudadanos, únicamente ofrece información sobre la tipología documental consultada y, así pues, debemos englobarlos como ciudadanos en general. Entre otras limitaciones, el tipo estudiante, atendiendo a criterios subjetivos, puede estar integrado tanto en el grupo de ciudadanos como en el de investigadores, dependiendo del grado de interés de su consulta, pero su adscripción al grupo de ciudadanos no podemos contabilizarla.

(6) En el estudio de usuarios internos, para ser riguroso, es necesario, además de posible, realizar entrevistas al personal; y, así, poder encuadrarlos en su estructura organizativa, cuantificarlos, conocer quiénes son usuarios potenciales y reales del archivo, sus hábitos, prioridades, sus problemas, el nivel de satisfacción, sus procedimientos... En fin, en este tipo de usuarios, la entrevista, como método directo de recogida de información, es fundamental; e incluso en el mismo momento de realizarse, ya podría establecerse un principio de acercamiento del archivo, en todos sus aspectos y niveles, al gestor administrativo. Por otro lado, sólo analizamos la relación del usuario interno con la documentación ya conservada, organizada y descrita que se custodia en el Archivo Municipal, no con la documentación que él mismo mantiene en su archivo de oficina. Pero también, como ejemplo de otros y múltiples aspectos a estudiar, podría analizarse la frecuencia con que los servicios administrativos consultan los documentos que custodian en sus propias oficinas para planificar, entre otras actuaciones, plazos de transferencia.

(7) En el plano teórico, igualmente útil para el posterior diseño del aplicativo, las actuaciones básicas han sido las de recopilación bibliográfica, clasificación, estudio y análisis de la misma.

(8) La herramienta informática que nos permite el vaciado de la información recogida y su posterior recuperación por campos aislados o entrelazados, es el gestor de bases de datos Access, que, de diferente manera, también se utilizó en el Estudio de Usuarios del Archivo Histórico Provincial de Zaragoza (Pla, 2000).

(9) Definir las características particulares de este tipo de usuario en el Archivo Municipal de Logroño requiere un estudio a fondo y pormenorizado con un nivel de análisis detallado.

(10) Esta familiarización es la que elimina el miedo del investigador. Su confianza le permitirá comunicarse abiertamente y formular preguntas directas a su entorno y al documento. Siguiendo la obra de Abraham H. Maslow, Motivation and Personality, Hugh Taylor (1984, p. 16) nos dice: "Nuestras necesidades fisiológicas deben ser las primeras que queden satisfechas $\mathrm{y}$, hasta que no lo son, dominan nuestros pensamientos, como ocurre cuando sentimos constantemente hambre. Vienen después las necesidades relativas a la seguridad, el orden y la eliminación del miedo. Una vez mitigado el miedo, podemos aceptar el amor y el efecto y reaccionar con una mayor confianza frente a los demás, a la vez que nos sentimos más seguros de nosotros mismos". 


\section{4}

(11) Un modelo es el "Borrador de encuesta a entregar en un archivo con el fin de medir el nivel de aceptación inicial por parte de los usuarios para implantar un programa de actividades de información y referencia" (Navarro, 2001, p. 186-187). Es un instrumento que sirve para medir el nivel de aceptación inicial del servicio de referencia de los archivos. Es un utensilio práctico del cual podemos extraer datos sensibles del usuario, la percepción que tiene del archivo y las expectativas al acceder a los documentos.

(12) En el Libro Registro de Ciudadanos del Archivo Municipal de Logroño queda registrada tanto la consulta de un ciudadano sin estudios, como, por ejemplo, la de un arquitecto que realiza una consulta ocasional.

\section{Referencias}

Alberch, R.; Boadas, J. (1991). La función cultural de los archivos. Bergara: IRARGI, Centro de Patrimonio Documental de Euskadi, 1991. 94 p.

Alberch Fugueras, Ramón; Cruz Mundet, José Ramón (1999). ¡Archívese! Los documentos del poder, el poder de los documentos. Madrid: Alianza Editorial, 1999. 203 p.

Alberch Fugueras, Ramón; Boix Llonch, Lurdes; Navarro Sastre, Natàlia; Vela Palomares, Susana (2001). Archivos y cultura: manual de dinamización. Gijón: Ediciones Trea, 2001. $174 \mathrm{p}$.

Berche, Claire (1982). La utilización de los archivos por el gran público (Trad. esp. de Archivum. 29 (1982) 113-123). // Walne, Peter (rec.). La administración moderna de archivos y la gestión de documentos: el prontuario RAMP. París: Unesco, 1985. 367-380.

Cruz Mundet, J. M. (1994). Manual de archivística. Madrid: Fundación Germán Sánchez Ruipérez, 1994. 400 p.

Díez de los Ríos San Juan, M ${ }^{a}$ Teresa (1996). El Departamento de Referencias del Archivo Histórico Nacional. Organización y funcionamiento. // Boletín de la ANABAD. 46: 1 (1996) 235-259.

Esteban Navarro, Miguel Ángel (1997). La técnica de gestión integral de la información: nuevas tendencias de la representación y la organización del conocimiento en los sistemas de información en las organizaciones. // García Marco, F. J. (ed.). Organización del Conocimiento en Sistemas de Información y Documentación: Actas del II Encuentro de ISKO-España. Zaragoza: Universidad de Zaragoza, Capítulo Español de la Sociedad Internacional para la Organización del Conocimiento, 1997. 285-299.

Fernández Gil, Paloma (1997). Manual de organización de archivos de gestión en las oficinas municipales. Granada: CEMCI, 1997. 220 p.

Franz, Eckhart G. (1981). Servicios de acceso y de referencias: información y orientación al usuario (Trad. esp. de International Conference of the Roum Table on Archives. Oslo, 1981). // Walne, Peter (rec.). La administración moderna de archivos y la gestión de documentos: el prontuario RAMP. París: Unesco, 1985. 306-320.

Gay Molins, Pilar (1995). Los usuarios y el proceso técnico de la descripción. // Ruiz Rodríguez, A. Ángel. Manual de archivística. Madrid: Síntesis, 1995. 39-66. 
Gómez Hernández, José A. (1996). Los usuarios. // Orera, Luisa. Manual de Biblioteconomía. Madrid: Síntesis, 1996. 229-245.

Gómez Hernández, José A. (coord.) (2000). Estrategias y modelos para enseñar a usar la información: Guía para docentes, bibliotecarios y archiveros. Murcia: Editorial KR, 2000. 290 p.

Hernández Salazar, P. (coord.) (1997). Seminario Latinoamericano sobre formación de usuarios de la información y los estudios de usuarios. México: Universidad Nacional Autónoma de México, 1997. 85 p.

Iturrate i Colomer, Germà (1993). Aproximació teòrica a la tipologia de l'usuari d'arxius. // Lligall. Revista Catalana d'Arxivística. 7 (1993) 77-87.

Legislación Básica (1982). Tomo III: Patrimonio artístico, archivos y museos. Madrid: Ministerio de Cultura, Secretaría General Técnica, 1982.

Mauri i Martí, Alfred (1995). La imatge de l'arxiu. // Lligall. Revista Catalana d'Arxivística. 9 (1995) 97-103.

Navarro Bonilla, Diego (2000). Informar en el archivo: características y recursos del servicio de referencia archivístico. // Actas de las VII Jornadas Españolas de Documentación: La Gestión del Conocimiento: retos y soluciones de los profesionales de la información. Bilbao: Servicio Editorial de la Universidad del País Vasco, 2000. 39-47.

Navarro Bonilla, Diego (2001). El servicio de referencia archivístico: retos y oportunidades. // Revista española de Documentación científica. 24: 2 (2001) 178-197.

Pérez Moreno, E. (1998). Formación de usuarios en archivos: una asignatura pendiente. // Boletín ACAL. 80 (1998). 25-27.

Pla Julián, A. I. (2000). Estudio de Usuarios del Archivo Histórico Provincial de Zaragoza. Trabajo inédito del Curso El sistema de información aragonés del Programa de Doctorado Sistemas de Información y Documentación del año 2000.

Roper, Michael (1982). El uso académico de los archivos (Trad. esp. de Archivum. 29 (1982) 27-39. // Walne, Peter (rec.). La administración moderna de archivos y la gestión de documentos: el prontuario RAMP. París: Unesco, 1985. 27-39.

Sanz Casado, E. (1994). Manual de estudios de usuarios. Madrid: Fundación Germán Sánchez Ruipérez, 1994. 288 p.

Taylor, Hugh A. (1984). Los servicios de archivo y el concepto de usuario: un estudio del RAMP. París: Unesco, 1984. 